\title{
INCIDENT REPORTING IN THE EDUCATIONAL SECTOR IN LATVIA
}

\author{
Antonina Jemeljanenko \\ University of Latvia, Latvia \\ Lubova Mackevica \\ Baltic International Academy, Latvia
}

\begin{abstract}
From the risk management perspective educational sector is exposed to a number of different risks which may result in serious incidents: student safety, research labs (e.g. chemical), food provision, new educational program introduction, change of methodology in teaching, budget allocation, selecting of co-operation partners, recruitment of teachers, accreditation process, power failure in educational institution, personal data leaks, and other. The cost of claims to education institution, not only financial, but also from a public image standpoint-can be significant. Educational institutions need to protect students, teachers, faculty, administration, support workers, contracted workers, community and their institution's reputation and budget. If educational institution's reputation is affected, a serious threat to future admissions, endowments, and financial strength is posed. Identifying and forecasting these risks via the incident reporting process is a common approach used by the risk managers in the corporate sector. Although the educational sector may face lower losses rather than the industrial sectors, effective incident management would help to insure avoiding losses and be prepared for any upcoming challenges. In the education institutions of Latvia this practice is not widespread: according to empirical research results only $1 / 3$ of all respondents confirmed that their educational institution use incident reporting as a tool for effective risk management. The aim of this study is to analyse the practice of incident reporting in other sectors, where it is successfully used, and establish applicability of these methods to the sector of education. Recommendations on how to effectively apply incident reporting to the daily routine in education management are provided as well.
\end{abstract}

Keywords: incident reporting, risk management, management of education, quality assurance, safety. 


\section{Introduction}

The concept of incident management was established, and in some cases even is regulated by law, in such industries like banking, healthcare, aviation, construction, rail, energy, manufacturing, maritime, etc. (Margaryan, Littlejohn, 2016; Canham, Jun, 2018; Staender, 2011). Incident management is needed in order to improve organisation's performance and internal control environment; it is important to know what particular incidents occur in the organisation to be able to identify weakness points and possible breaches (Chapelle, 2019). Incident management practices in different industries were researched in the course of this study by analysing published scientific articles of other researchers in order to draw lessons how incident management could be applied in the sector of education. In parallel, an empirical research was performed to establish current usage of incident reporting in the educational institutions in Latvia by way of online questionnaire to the leaders of educational institutions. This study looks into how incident management could be applied to the sector of education, where currently this practice is not well known according to the results of performed empirical research. The conclusion is drawn taking into account the findings of desktop and empirical research results. In this study authors define incident as an event that is different from regularly followed routine due to failure in process or IT system, human factor errors or external environment caused situation.

\section{Methodology}

The study was conducted in two parts. First part consisted of systematic literature review research method. The search was performed in the Web of Science scientific database, entering the following key words in the search engine: "incident reporting", "incident management" and "incident management and reporting in education". Results of the search were analysed on the validity for research purpose and the most relevant articles for the last five years were selected for profound analysis and development of recommendations. In addition, relevant academic books published within the last year available to authors were reviewed for additional information related to the research. This research provided with comprehensive information about international practice of incident reporting and management, its pros and cons and the conditions required for successful implementation of incident reporting in different types of organisations, studied in more details in the following sections.

Second part of the research consisted of empirical research. Online questionnaire developed as part of wider research about risks management 
in the educational sector of Latvia, was partially used for this research, where respondents, apart from other risk management questions, were asked to answer whether the education institution to which they belong, performs collection and registration of incidents. The question was structured as multiple choice with the following options:

1. Yes, incidents are registered in the risk database;

2. No, incidents are not registered;

3. I do not know;

4. Please specify if neither of the options applies (space was provided for the comment).

Questionnaire was placed into online platform SurveyMonkey.com generating online link and allowing participants to stay anonymous. Link to the survey was distributed via social networks, e-mail to the Association of Education Leaders in Latvia and e-mailed to 739 e-mail addresses of various educational institutions of Latvia (secondary schools, colleges, universities, pre-school institutions). In addition, 10 printed questionnaires were distributed to students of Pedagogy and Psychology Faculty at the premises of the University of Latvia.

Following qualitative review of returned questionnaires, it was established that a total of 127 stakeholders fully completed and returned the questionnaire in good quality; all of them have direct relation to educational sector from different parts of Latvia - majority were managers and leaders of educational institutions, most of the responses were received from secondary schools. For this paper, only one question relating to incident reporting was used.

\section{Results}

Occurrence of incidents is unavoidable and even a minor incident can provide valuable feedback for improvement of safety, quality and management in the organisation (Westhuizen, Stanz, 2017; Staender, 2011). Staender (2011), Margaryan, Littlejohn, (2016) and Stemn, Bofinger, (2018) discuss the concept of "learning from incidents", which means that the organisation can learn from unexpected safety events to prevent similar such events that may happen in the future. This may be applicable not only to safety events, but any incidents, that cause any kind of losses. Nowadays the concept is applied in variety of industries, such as manufacturing, aviation, healthcare, transport, banking and finance, etc. (Margaryan, Littlejohn, 2016; Staender, 2011). Incident reporting provides the opportunity to reveal the weaknesses that allowed incident to happen and to act upon it to make required improvements (Staender, 2011; Chapelle, 2019). 
The effective process contains important steps, which include reporting of incident, thorough investigation to identify and analyse the root causes of the incident and then drawing out recommendations and "learning points", that are used for implementation of required changes in order to prevent the same incident from re-occurring (Staender, 2011; Margaryan, Littlejohn, 2016; Stemn, Bofinger, 2018). Staender (2011) compares incident reporting to the storytelling and states that these reports can be collected by an organisation as documented experience used for applying necessary improvements.

Another important step in incident management is to communicate the required changes in human behaviour or processes to relevant responsible stakeholders, as without good communication implementation of changes (or mitigation actions) will not be effective and may not take place at all, if not addressed to the key responsible people in the organisation. The communication channels can be reports, internal regulations and manuals for employees, notice boards, notification e-mails, team discussions (Margaryan, Littlejohn, 2016). Stemn, Bofinger (2018) noted that learning from incidents is the key for achieving high levels of safety in organisations. Moreover, incident reporting is useful for scenario planning and analysis, which should be used for critical incident planning and management. Incident database can serve as a great input for risk modelling and scenario planning (Mazaheri, Montewka, 2015; Chapelle, 2019).

Figure 3 reflects the results provided by the empirical research question related to incident reporting in educational institutions of Latvia. As it can be seen, only $32 \%$ of respondents provided confident answer that incidents are recorded in their educational institution. Another $9 \%$ specified that not all, but several cases that meet certain criteria (e.g. serious trauma caused to student at school that result in calling an ambulance, school closure due to emergency situation, etc.) are recorded in internal register. $27 \%$ of respondents confirmed that in their educational institutions there is no incident reporting routine and 32\% responded that they do not know whether incidents are recorded, what means that incident reporting awareness is not practiced in their institutions and therefore it is assumed that incidents are not recorded in their institutions, as the key prerogative for incident reporting and management is awareness of employees about such requirement. In addition, ten respondents, provided following comments below the question:

- "We have incident register, but nothing is registered in it"

- "Never heard about incident reporting"

- "Did not have any need for incident reporting"

- "We create an act about what has happened"

- "Never had any incidents" 
- "Incidents are registered according to status (students, teachers, etc.)"

- "Incident are partially registered"

- "Incidents are registered if needed"

- "Acts are made about work related incidents"

- "Protocols and acts are made for incidents"

Hence, empirical research results demonstrated quite low awareness of respondents about incident reporting and low usage of this management tool in the educational institutions of Latvia. It is sometimes replaced with formal protocols and acts, as provided by some comments.

\section{Does the educational institution to which you belong perform collection and registration of realised incidents in the internal incident or risk database?}

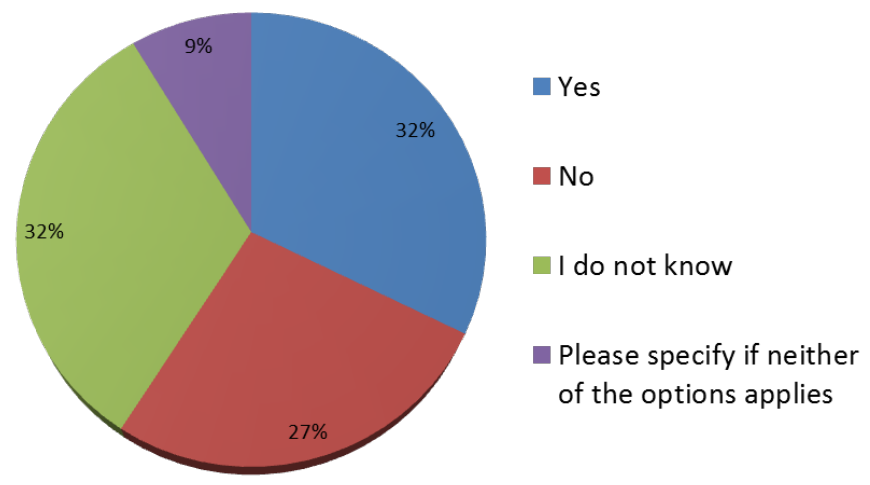

Figure 1. Distribution of answers on incident reporting routine question

\section{Discussion}

Studies of Margaryan, Littlejohn (2016) and Westhuizen, Stanz (2017) identified that there are problems with behaviour of reporting, i.e. incidents often are not reported because employees are reluctant to report due to the burden of completing incident report and consequent liability to get involved in the investigation or root causes. Lack of understanding of the incident causes or the importance of the incidents is additional reasons for avoiding reporting incidents. Also often organisational ethics act as the key driver for reporting or not reporting the incidents (Westhuizen, Stanz, 2017). 
Filtness, Goode (2015) performed a research about usage of incident reporting system in hot air ballooning industry and came to conclusion that majority of respondents did not report incidents mainly due to lack of understanding of incident reporting process, when to report and how, as well as not seeing the significance of incidents to be reported. Another important aspect raised by majority of researchers is ease of incident reporting process: the simpler is the routine the more successful it becomes. Westhuizen, Stanz (2017) highlighted the perceived burden of incident reporting, which served as predicament for active reporting of incidents in air navigation industry. Hence, user-friendly simple system is an important factor in successful implementation if incident reporting in any organisation.

Staender (2011) in addition mentions the fear for reporting, as it may affect reporter's further career. Filtness, Goode (2015) mention incident database as an important tool for holistic incident management for implementation of improvements and follow-up. In addition, aspects of privacy and usefulness are important, as often the reporters do not want to be recognised and there should be value in spending time into the reporting of incidents, what means that it is expected that the incident will be investigated, followed up and conclusions for improvements will be drawn.

There are a number of different methodologies how incidents can be analysed, lessons learned applied and discovered risk effectively managed. Stemn, Bofinger (2018) highlight bowtie analysis (BTA) as the most effective tool for learning from incidents, where the focus is on threats, consequences and required mitigation controls to prevent the threats from realising and to minimise the consequences. By focusing on the threats, it is possible to develop mitigation action plans that will control threats and prevent the incident, whereas by focusing on consequences, mitigation actions will minimise the impact of realised threats.

Canham, Jun (2018) in turn discuss the usability of Systems-Theoretic Accident Model and Processes (STAMP) concept, which consists of 8 key steps:

1. identification of threats related to incident

2. identification of safety-related impediments related to the incident

3. documentation of safety controls in place ought to control the threats

4. determining possible events that lead to the incident and analysing frontline controls

5. analysing higher-level controls and reasons why they allowed threats to realise and how

6. studying overall communication and management problems that could have led to the incident 
7. looking for any recent changes that could have contributed to weakening of controls and contributed to the incident

8. development of recommendations and mitigation action plans to strengthen controls and prevent incident from re-occurring in the future. This approach is very comprehensive and being applied would provide very comprehensive risk picture to the organisation, however its disadvantage is in the amount of required time and effort, what most of the organisations would not be able to contribute in order to fulfil the entire process.

Staender (2011) in his study discussed so-called "plan-do-check-act" (PDCA) cycles, concerned with the incidents that call for initiating of improvement process. This is simpler and less time-consuming approach, where it is possible to act upon the incident by planning concrete mitigation actions, implementing then and then performing follow-up on the effectiveness of controls. This is the most popular approach applied by the organisations due to its simplicity and effective use of resources. In combination with BTA approach discussed above, it would be possible to focus planned actions on threats or on consequences and thus mitigate the most painful problems that organisation is facing.

Stemn, Bofinger (2018) researched the consequences of not using incident reporting as a tool for learning and improving organisational performance and came to the following conclusion, that not paying attention to incident recording and learning from incidents leads to:

- regular repetition of similar incidents that wastes time and resources on frequent resolution of the same problem

- transformation of insignificant incidents into significant and even crisis

- incidents start to be accepted as normal course in daily routine, thus affecting long-term quality of provided services.

Stemn, Bofinger (2018) highlight that the key prerequisite for successful implementation of learning from incidents is internal culture focused on learning and self-improvement. In addition, organisations need to ensure qualitative collection of incidents and root causes, thorough investigation and developing efficient and well communicated improvement plan. According to Staender (2011), prerequisites for successful implementation of incident reporting are the following important factors: comprehensive training of what should be reported and how, continuing trainings on the importance and purpose of incident reporting, criteria for reporting, list of incident examples, user-friendly reporting form, clarity on how to report, dedicated person allocated for reported incident management, regular feedback on reported incidents, etc. In addition, Staender (2011) states, that an important aspect is ensuring confidentiality of the person reporting 
an incident and making sure that the information will not be used against them.

The following type of register can be used by the educational institutions to record all incidents that happen, including low impact incidents. This type of register would be easy to use and the questions would guide the reporter on what needs to be recorded, what will subsequently assist in more comprehensive analysis if needed:

Table 1. Incident reporting form example

\begin{tabular}{|l|l|}
\hline Incident nr. & $\begin{array}{l}\text { It is important to allocate specific number for each } \\
\text { separate incident }\end{array}$ \\
\hline What happened? & Short description of incident \\
\hline $\begin{array}{l}\text { When and How was it } \\
\text { discovered? }\end{array}$ & $\begin{array}{l}\text { Short description how the incident was discovered to } \\
\text { reveal transparency or any breaches }\end{array}$ \\
\hline What are the root causes? & $\begin{array}{l}\text { Most important part to analyse why the incident } \\
\text { happened and what caused it }\end{array}$ \\
\hline $\begin{array}{l}\text { What are the } \\
\text { consequences/losses? }\end{array}$ & $\begin{array}{l}\text { Description of financial and non-financial losses and } \\
\text { consequences }\end{array}$ \\
\hline When and how did it start? & $\begin{array}{l}\text { Important to note to better understand root causes and } \\
\text { possible additional breaches }\end{array}$ \\
\hline $\begin{array}{l}\text { What mitigation actions } \\
\text { are planned/executed? }\end{array}$ & $\begin{array}{l}\text { Here the focus can be on mitigating the root cause or the } \\
\text { consequences }\end{array}$ \\
\hline Status & $\begin{array}{l}\text { Status of the mitigations actions and the incident itself, } \\
\text { e.g. "closed, mitigation actions no needed", or "open, } \\
\text { mitigation actions in process", or "closed, mitigation } \\
\text { actions in process", etc. }\end{array}$ \\
\hline
\end{tabular}

Threshold for reporting should not be set in order not to miss small but frequent events that impact the quality of education and general risk profile. Incidents can be recorded in a journal one by one or placed in Excel database with the same columns made vertical for recording many incidents on one sheet in order to have holistic summary for analysis and reporting.

\section{Conclusion}

Incident reporting is an important tool for learning from own failures and for effective management. In the sector of education, where the main stakeholders are children, incident reporting is crucial, as it helps to strengthen security and safety, improves the quality of education and provides holistic risk picture for each individual institution and for groups 
of institutions, addressing their current problems. Having an incident register in every educational institution, which is filled in from completed incident reporting templates, as provided in Table 1, would assist managers and officials to address correct problems faced by concrete institutions and to analyse regional problems and correlation of incidents with other factors that would help to realise required developments. Incident register provides managers with holistic overview of the risk picture and thus adds value to more informed decision making and budget planning.

Nevertheless, it is needed to better understand how to motivate educational institutions to adapt the practice of incident reporting and how to make this tool more popular and useful. Hence additional research is needed in way of interviewing stakeholders in the educational sector in order to better understand struggles and predicaments faced by educational sector managers and employees in regards to incident reporting tools and incident management.

\section{References}

Canham, A., Jun G. T., Waterson, P., Khalid, S. (2018) Integrating systemic accident analysis into patient safety incident investigation practices, Applied Ergonomics, V. 72 (2018), pp. 1-9, retrieved from Web of Science scientific database on 24.112018

Chapelle, A. (2019) Operational Risk Management, UK: John Wiley \& Sons, Ltd.

Filtness, A. J., Goode, N., Cook, R. (2015) Incident reporting culture in recreational hot air ballooning, Procedia Manufacturing, V.3 ( 2015 ) pp. 1165-1172, retrieved from Web of Science scientific database on 24.112018

Margaryan, A., Littlejohn, A., Stanton, N. (2016) Research and development agenda for Learning from Incidents, Safety Science Journal, V. 99 (2017), p. 5-13, retrieved from Web of Science scientific database on 24.112018

Mazaheri, A., Montewka, J., Nisula, J., Kujala, P. (2015) Usability of accident and incident reports for evidence-based risk modeling - A case study on ship grounding reports, Safety Science, V. 76 (2015), pp. 202-214, retrieved from Web of Science scientific database on 24.112018

Staender, S. (2011) Incident reporting in anaesthesiology, Best Practice \& Research Clinical Anaesthesiology, V. 25 (2011), pp. 207-214; retrieved from Web of Science scientific database on 24.112018

Stemn, E., Bofinger, C., Cliff, D., Hassall, M. E. (2018) Failure to learn from safety incidents: Status, challenges and opportunities, Safety Science, V. 101 (2018), pp. 313-325, retrieved from Web of Science scientific database on 24.112018

Westhuizen, J., Stanz, K. (2017) Critical incident reporting systems: A necessary multilevel understanding, Safety Science, V. 96 (2017), pp. 198-208, retrieved from Web of Science scientific database on 24.112018 